\title{
Marine Strata Stochastic Spectral Response
}

\author{
WANG Ya Jun \\ School of Maritime and Civil Engineering. \\ Zhejiang Ocean University \\ Zhoushan, China \\ aegis68004@163.com
}

\begin{abstract}
The dynamic shear rigidity is an important function for marine soil. Due to the random fluctuation, the proper expression on marine soil dynamic shear rigidity is stochastic spectral theory. The typical marine sediments were studied here. Their dynamic characteristics were included systematically in this paper. Some key dynamic functions were established based on physical-mechanical tests for the marine sediments. Furthermore, the primary spectral functions were established here based on FFT and the stochastic spectral characteristics were offered. The analysis results show that marine strata are possessed of random mechanical nature. These results in this paper are helpful for both marine structures application and academic researches.
\end{abstract}

Keywords-dynamic tests; dynamic shear rigidity; darine strata; stochastic spectral response; FFT

\section{INTRODUCTION}

Marine environmental engineering cases confront uncertain wave loading influence1,2. Particularly, there lives an urgent demand for super road nets alongside the Chinese coastline. The marine roadbed strata here are born with low strength. Under the random traffic loading conditions, the expectation of maximal wave-height Zhoushan Seas reaches $10 \mathrm{~m}$. Most marine structures inclusive of super road nets are threatened by the fluctuation of stochastic wave which holds great momentum3,4,. Especially, most fixed structures are controlled by the variation of marine strata where is the foundation of them. However, the shortage of marine soil parameters incurs unreliability of structures working behavior. At the same time, most marine roadbed strata of Zhoushan Seas are born with the stochastic characteristics of these geo-mechanical parameters.

Zhoushan Seas lives in East China Sea. The shoreline here, extending $2444 \mathrm{~km}$, consist chiefly of marine deposits. The local marine strata are born with highly complex chemical-physical characteristics and mechanical behavior. Due to overdevelopment as well as arbitrary urbanization, the regional bio-geological configuration has been disturbed heavily. Moreover, Zhousha Seas approach Sino Jiangshao plate around which the neighboring plates came into domino active period with its singular stability. Meanwhile, the high-spreading reclamations produced massive dredger fills that aggravated the mechanics qualities of marine strata. These facts undermined the regionally geological safety.

\section{PRIMARY StOchastic SPECTRAL CHARACTERISTICS OF ZHOUSHAN MARINE STRATA}

Under the bidirectional exciting loading condition, the dynamic shear rigidity of marine soil can be computed as Eq. 1.

$$
G_{d_{i}}=\frac{\Delta \bar{\sigma}_{i}}{\Delta \varepsilon_{d_{i}}}
$$

where, $G_{d_{i}}$ designates the value of dynamic shear rigidity under No. i cycle loading; $\Delta \bar{\sigma}_{i}$ and $\Delta \varepsilon_{d_{i}}$ represent the increment of dynamic stress and strain under No. i cycle loading.

According to the spectral figure (Figure 1.), the marine soil rigidity spectrum is energy spectrum whose numerical domain has explicit margin. Due to the fluctuation of softening and hardening process of marine soil, its energy spectrum is negative-positive dyadic spectrum.

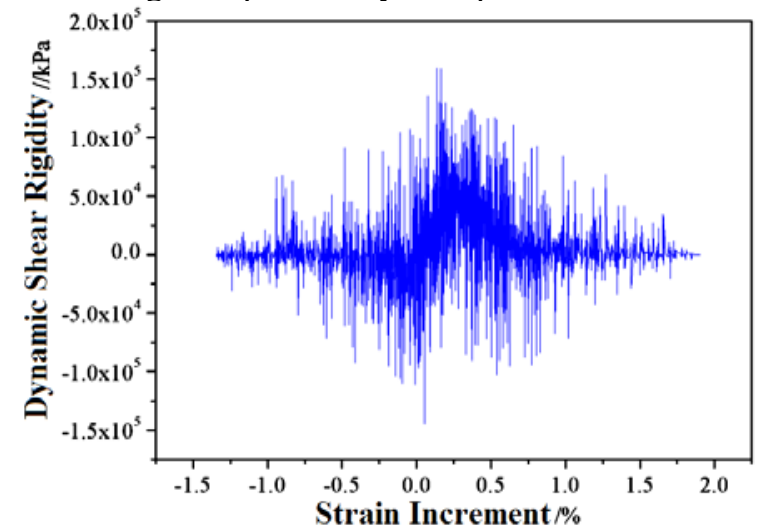

Figure 1. Dynamic Shear Rigidity

According to the distribution characteristics of Zhoushan marine soil, the stochastic fluctuation of its rigidity can adopt fractional random signal model (Fig. 1 and Fig. 2). The envelope line is showed in Eq. 2. So, the stochastic dynamic shear rigidity signal can be deduced by Eq. 3 .

$$
g\left(\varepsilon_{d}\right)=e^{\varepsilon_{d}+13.4} \quad\left\{g \geq 0,-5.379 \% \leq \varepsilon_{d} \leq-1.4163 \%\right\}
$$

where, ${ }^{\varepsilon_{d}}$ is dynamic strain; g designates the stochastic signal envelope function. 


$$
\begin{gathered}
G\left(\varepsilon_{d}\right)=e^{\varepsilon_{d}+13.4} \cdot \sin \left[\pi \frac{\left(\varepsilon_{d}+3.3\right)^{6}+0.01}{1.001}\right] \\
\left\{-5.379 \% \leq \varepsilon_{d} \leq-1.4163 \%\right\}
\end{gathered}
$$

where, $\mathrm{G}$ represents stochastic dynamic shear rigidity signal model.

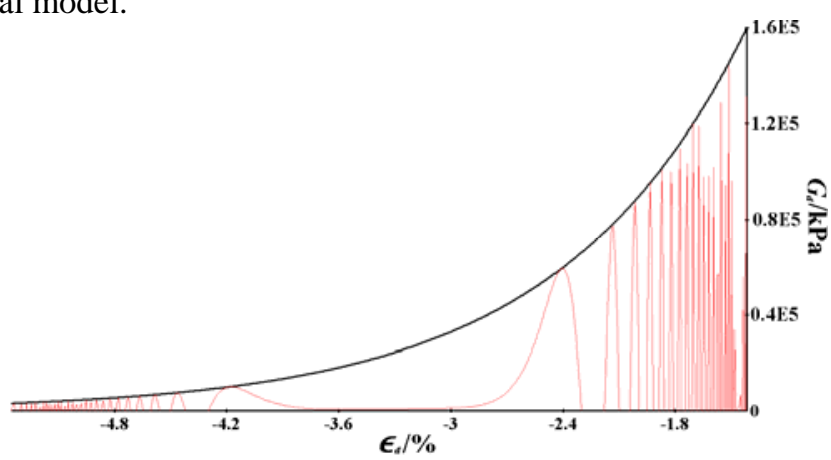

Figure 2. Stochastic Signal of Fractional Dynamic

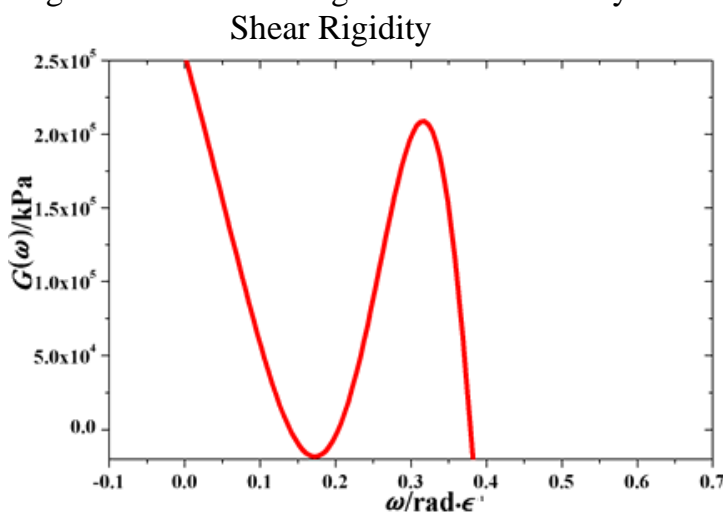

(a)

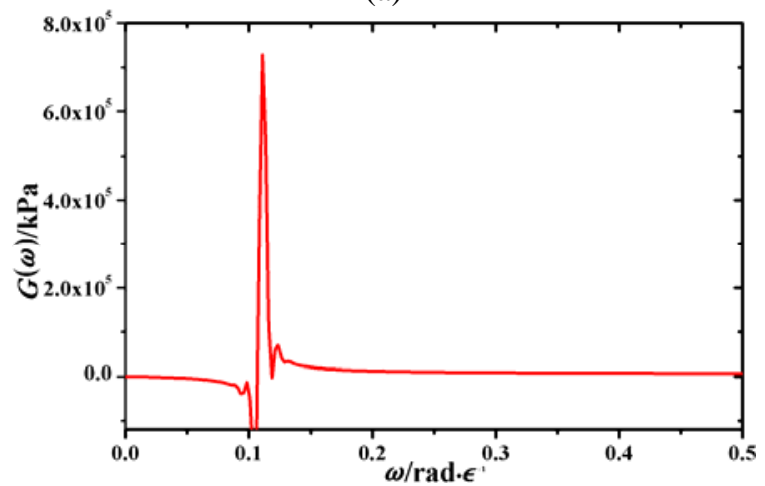

(b)

Figure 3. Stochastic Dynamic Shear Rigidity Spectrum

$G(\omega)=G_{0}+A_{G}\left[m_{u} \frac{2}{\pi} \frac{w}{4\left(\omega-\omega_{c}\right)^{2}+w^{2}}+\left(1-m_{u}\right) \frac{\sqrt{4 \ln 2}}{\sqrt{\pi} w} e^{-\frac{4 \ln 2}{w^{2}}\left(\omega-\omega_{c}\right)^{2}}\right]$

The stochastic dynamic shear rigidity spectrum (Fig. 3) can be computed by Eq. 4 based on FFT technology. where, $G_{0}=772504.37 \mathrm{kPa}, A_{G}=-1.173 \times 10^{6} \mathrm{kPa}$, $\omega_{c}=0.453, w=0.306, m_{u}=2.11$.

All of them are the fitted parameters for stochastic dynamic shear rigidity spectrum.
For application, the amplitude of spectral model is very important $[5,6]$. The probabilistic distribution of stochastic dynamic shear rigidity amplitude (Fig. 4) is expressed in Eq. 5 based on statistical simulation.

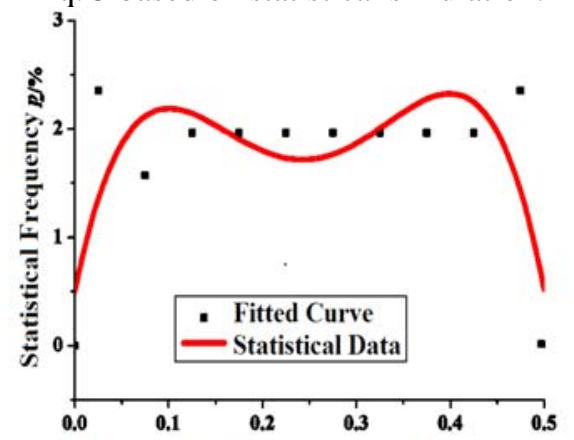

Frequency of Stochastic Dynamic Shear Rigidity Spectrum $\omega /$ rade Figure 4. Probabilistic Distribution of Stochastic Dynamic Shear Rigidity Amplitude

$$
\begin{aligned}
& f_{p}(\omega)=p_{0}+p_{1} \times \omega+p_{2} \times \omega^{2}+p_{3} \times \omega^{3}+p_{4} \times \omega^{4} \\
& 0 \leq \omega \leq 0.5
\end{aligned}
$$

where, $p_{0=0.507}, p_{1=42.011}, \quad p_{2=-348.125}$,

$p_{3=1067.458,} p_{4=-1078.237}$. All of them are the fitted parameters for probabilistic distribution[7,8].

So, the dynamic shear rigidity amplitude function can be expressed in Eq. 6 whose distribution is showed in Fig. 5.

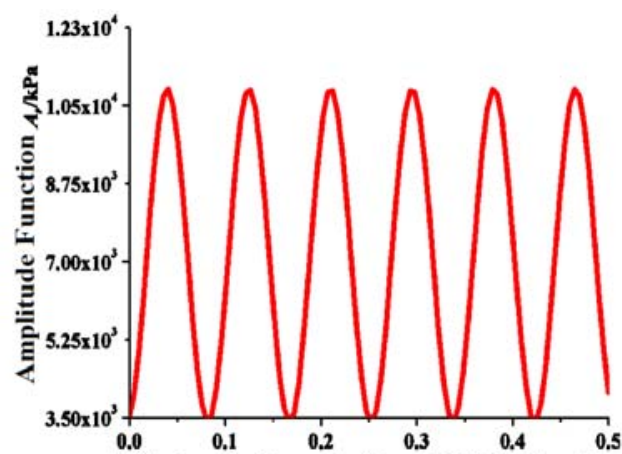

Frequency of Stochastic Dynamic Shear Rigidity Spectrum $\nsim /$ rad $\epsilon^{\prime}$ Figure 5. Dynamic Shear Rigidity Amplitude Function

$$
A_{p}(\omega)=a_{p_{0}}+a_{p_{1}} \sin \left(\pi \frac{\omega-a_{p_{2}}}{a_{p_{3}}}\right)
$$

where, $\quad a_{p_{0}}=7140.63 \mathrm{kPa}, \quad a_{p_{1}=3741.22 \mathrm{kPa}}$, $a_{p_{2}=0.018,} a_{p_{3}=0.043 \text {. All of them are the fitted }}$ parameters for dynamic shear rigidity amplitude function$[9,10]$.

\section{DYNAMIC HISTORY MODEL}

Due to the significant randomness of marine soil strength under cyclic loading conditions, the establishment of random dynamical strength spectrum is one crucial conception to disclose the random fluctuation of marine soil strength. First, damping sine wave, according to the relation curve on deviatonic-stress and 
loading duration, is used to simulate dynamical strength time-history curve based on soil strength dissipation during the bidirectional vibration. The time-history function is founded as Eq. (7).

$$
S(\tau)=A_{S} \times e^{\left(-\frac{\tau^{a_{0}}}{t_{0}}\right)} \times \sin \left[\pi \frac{\left(\tau-\tau_{c}\right)}{w}\right] \text { (7) }
$$

The dynamical strength time-history function consists of three parts: $A_{s}$ designates dynamical strength amplitude; negative exponential item represents energy dissipation based on stationary probabilistic density; harmonic item indicates damping effect. There are 5 undetermined coefficients in this function. The fitted values for MS1 are:

$$
\begin{aligned}
& A_{s}=62 \mathrm{kPa}, \quad a_{0}=1.3 \quad, \quad t_{0}=577.87, \\
& \tau_{c}=-0.01247, \quad w=1.00123
\end{aligned}
$$

The dynamical strength time-history on marine stratum is showed in Figure 6.

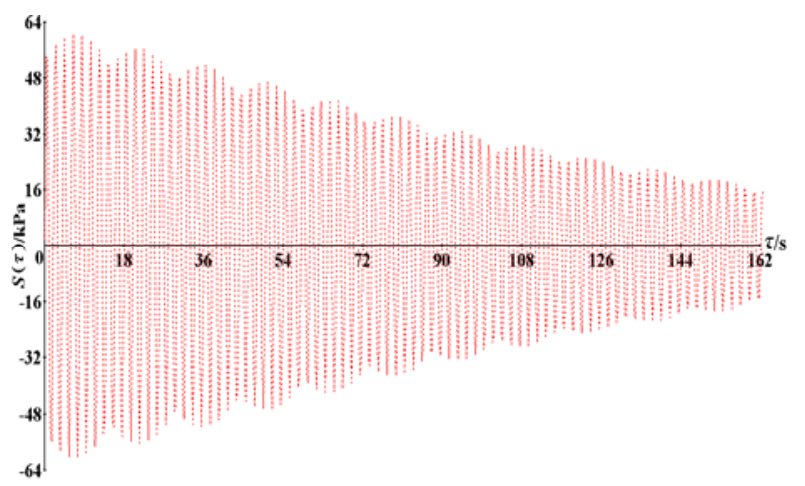

Figure 6. Dynamic History Curve

According to the random spectrum the characteristics of dynamic strength of zhoushan marine sand, the corresponding order moments of can be obtained. The most valuable is the first order moment, it can be used as usually digital features which is used in engineering analysis and calculated according to formula (8).

$$
\begin{aligned}
& E(\theta, S(\omega))=\int_{0}^{\theta} \omega S(\omega) d \omega \\
& =\int_{0}^{\theta} \omega\left\{\left\{S_{\omega}+\frac{A_{\omega}}{\kappa \sqrt{\pi / 2}} \times e^{\left[-2\left(\frac{\omega-\omega_{c}}{\kappa}\right)^{2}\right]}\right\} \times \cos \left(a_{1}-\frac{a_{2}}{1+e^{\frac{\omega-\omega_{0}}{\zeta}}}\right)\right\} d \omega
\end{aligned}
$$

where, $\theta=0.5 \mathrm{rad} / \mathrm{s}$ is the exciting frequency of marine stratum. With the exciting frequency of marine stratum, the mean frequency of it can be computed as

$$
\bar{\omega}_{s}=\frac{E(\theta, S(\omega))}{\int_{0}^{\theta} S(\omega) d \omega}
$$

The result of the mean frequency of marine stratum in Zhoushan is $\bar{\omega}_{s}=0.16 \mathrm{rad} / \mathrm{s}$
According to the limit of energy of soil, the stochastic dynamic rigid spectrum can be expressed as

$$
\begin{aligned}
& A(\omega)=\frac{\int_{\varepsilon_{\text {dint }}}^{\varepsilon_{d \text { app }}} g\left(\varepsilon_{d}\right) \cos \left(\omega \varepsilon_{d}\right) d \varepsilon_{d}}{\int_{\omega_{\text {nif }}}^{\omega} A_{p}(\varpi) f_{p}(\varpi) d \varpi}
\end{aligned}
$$

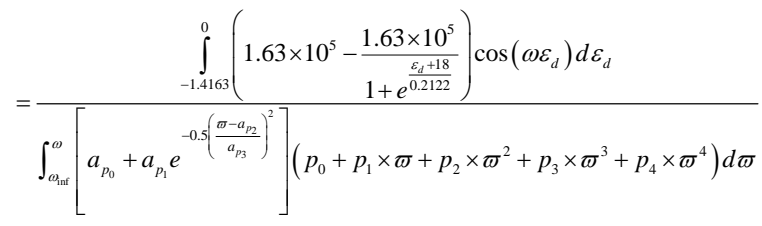

The power series can be computed as

$$
\begin{aligned}
& \int_{-1.4163}^{0}\left(1.63 \times 10^{5}-\frac{1.63 \times 10^{5}}{1+e^{\frac{\varepsilon_{d}+18}{0.222}}}\right) \cos \left(\omega \varepsilon_{d}\right) d \varepsilon_{d} \\
= & \int_{-1.4163}^{0}\left(1.63 \times 10^{5}-\frac{1.63 \times 10^{5}}{1+\left[6.91 \times 10^{36} \times\left(1+\varepsilon_{d}+\frac{\varepsilon_{d}^{2}}{2 !}+\cdots\right)\right]}\right]\left[1-\frac{\left(\omega \varepsilon_{d}\right)^{2}}{2 !}+\frac{\left(\omega \varepsilon_{d}\right)^{4}}{4 !}\right] d \varepsilon_{d} \\
\approx & \int_{-1.4163}^{0} 1.63 \times 10^{5} \times\left[1-\frac{\left(\omega \varepsilon_{d}\right)^{2}}{2 !}+\frac{\left(\omega \varepsilon_{d}\right)^{4}}{4 !}\right] d \varepsilon_{d}
\end{aligned}
$$

With approximation, it can be simplified as

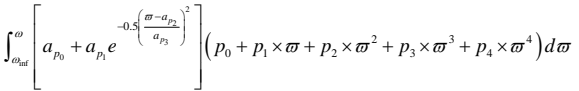

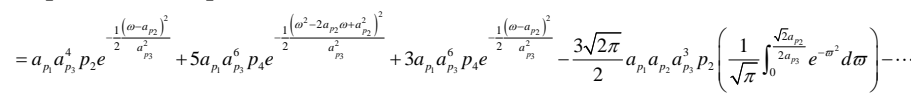

$$
\begin{aligned}
& \frac{3 \sqrt{2 \pi}}{2} a_{p_{1}} a_{p_{2}} a_{p_{3}}^{3} p_{2}\left(\frac{1}{\sqrt{\pi}} \int_{0}^{\frac{\sqrt{2}\left(\omega-a_{p_{2}}\right)}{2 a_{p_{3}}}} e^{-\sigma^{2}} d \sigma\right)+\frac{\sqrt{2 \pi}}{2} a_{p_{1}} a_{p_{2}}^{2} a_{p_{3}} p_{2} \omega\left(\frac{1}{\sqrt{\pi}} \int_{0}^{\frac{\sqrt{2}\left(\omega-a_{\left.p_{2}\right)}\right.}{2 a_{p_{3}}}} e^{-\sigma^{2}} d \sigma\right)-\cdots \\
& \frac{3 \sqrt{2 \pi}}{2} a_{p_{1}} a_{p_{3}}^{5} p_{3}\left(\frac{1}{\sqrt{\pi}} \int_{0}^{\frac{\sqrt{2}\left(\omega-a_{\left.p_{2}\right)}\right.}{2 a_{p_{3}}}} e^{-\sigma^{2}} d \sigma\right)+\frac{3 \sqrt{2 \pi}}{2} a_{p_{1}} a_{p_{3}}^{5} p_{4} \omega\left(\frac{1}{\sqrt{\pi}} \int_{0}^{\frac{\sqrt{2}\left(\omega-a_{p_{2}}\right)}{2 a_{p_{3}}}} e^{-\pi^{2}} d \sigma\right)-\cdots \\
& \frac{\sqrt{2 \pi}}{2} a_{p_{1}} a_{p_{2}}^{5} a_{p_{3}} p_{4}\left(\frac{1}{\sqrt{\pi}} \int_{0}^{\sqrt{2}\left(\omega-a_{\left.p_{2}\right)}\right.} e^{-a_{p_{3}}} e^{-\omega^{2}} d \sigma\right)+3 \sqrt{2 \pi} a_{p_{1}} a_{p_{2}}^{2} a_{p_{3}}^{3} p_{4} \omega\left(\frac{1}{\sqrt{\pi}} \int_{0}^{\frac{\sqrt{2}\left(\omega-a_{p_{2}}\right)}{2 a_{p_{3}}}} e^{-\omega^{2}} d \sigma\right)+\cdots
\end{aligned}
$$

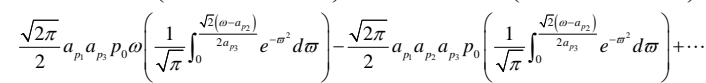

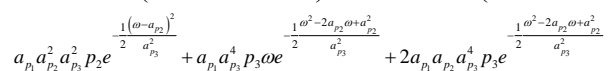

Based on the calculation above, the stochastic signal can showed in Figure 7.

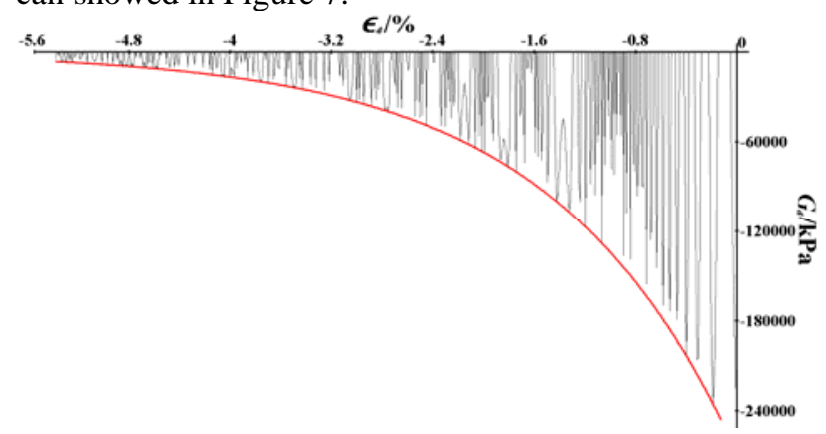

Figure 7. Stochastic Signal curve on marine stratum

\section{CONCLUSIONS}

With stochastic spectral theory, the highly complicated mechanical characteristics of Zhoushan marine strata can be deduced comprehensively. Particularly, these stochastic spectral parameters and functions are helpful 
for quantification of marine structures' design and analysis that are influenced by such complex dynamical forces as bidirectional exciting loading etc.

\section{ACKNOWLEDGEMENTS}

This work was supported by the National Natural Science Foundation of China (Grant No: 51109118), Zhejiang Provincial Natural Science Foundation of China (Grant No: LY14E090001), United Development Project Foundation from Zhejiang Ocean University and Wenzhou University(Grant No: 21188004113) , United Development Project Foundation from Zhejiang Ocean University and Hydrochina Huadong Engineering Corporation (Grant No: 21188004013) and Young Teachers Improvement Project Fund of Zhejiang Ocean University (Grant No: 11042101512).

\section{REFERENCES}

[1] Y. J. Wang, W. H. Zhang, C. H. Zhang and F. Jin, "Fuzzy Stochastic Damage Mechanics (FSDM) Based on fuzzy auto-adaptive control theory,” WSE. Vol.5, 2, pp.230-242, 2012.

[2] Y. J. Wang, W. H. Zhang, W. L. Jin, C. Y. Wu and D. C. Ren, "Fuzzy Stochastic Generalized Reliability Studies on Embankment Systems Based on First-order Approximation Theorem", WSE. Vol.1, 4, pp.36-46, 2008.
[3] Y. J. Wang, W. H. Zhang, W. L. Jin, C. Y. Wu and D. C. Ren, "Stochastic Seepage Field Studies on Main Embankment of Yangtse Rive in Southern Jingzhou Zone of China”, Manufacturing Science and Technology, Vol.295,pp. 2422-2429, 2011.

[4] Y. J. Wang, W. H. Zhang, C. Y. Wu and D. C. Ren, "Safety Model Comprehensive Judgement Application for Dike Construction Risk Evaluation System Research”, Manufacturing Science and Technology. Vol.295, pp. 2345-2352, 2011.

[5] Y. J. Wang, W. H. Zhang, "Rock Slope Fuzzy Stochastic Damage Study”, Advanced Materials Research. Vol. 524-527, pp. 337-340, 2012.

[6] Y. J. Wang, W. H. Zhang, "Super Gravity Dam Generalized Damage Study”, Advanced Materials Research. Vol. 479-481, pp. 421-435, 2012.

[7] Y. J. Wang, "Application of generalized reliability method based on fuzzy-stochastic theorem in bank slope stability analysis", Geo Eng Tech, Vol. 18, no. 5, pp. 217-223, 2004.

[8] Y. J. Wang, C. Y. Wu. 2014. Uncertainty on geo-engineering. Science Press of China.

[9] Y. J. Wang, W. H. Zhang. "Studies on non-linear fuzzy stochastic damage field”. Journal of Hydraulic Engineering, Vol.41, no.2, pp.189-197, 2010.

[10] Y. J. Wang. "Primary physical-mechanical characteristics on marine sediments from Zhoushan Seas in Sino mainland". Applied Mechanics and Materials, Vol. 275-277, pp.273-277, 2013. 